\title{
Genetic incompatibility combined with female-lethality is effective and robust in simulations of Aedes aegypti population control
}

Maciej Maselko, Stephen Heinsch, Siba Das, and Michael J. Smanski

Department of Biochemistry, Molecular Biology, and Biophysics and BioTechnology Institute. University of Minnesota - Twin Cities, Saint Paul, MN, 55108, USA

Correspondence and requests for materials should be addressed to M.J.S. (smanski@umn.edu). 
Recent reports of CRISPR/Cas9-based suppression gene drives in insects underscore the challenge of overcoming genetic resistance. Here we present results from agent-based simulation modeling of a novel Field-Amplified Male Sterility System (FAMSS) that outperforms suppression gene drives when challenged with genetic resistance. FAMSS combines a recently described synthetic genetic incompatibility approach with previously demonstrated female-lethality constructs. Our results suggest that FAMSS will be an effective strategy for temporally and spatially self-limited suppression of the disease vectoring mosquito, Aedes aegypti. 


\section{Introduction}

Genetically engineered (GE) biocontrol agents can provide effective, species-specific population suppression of disease vectors ${ }^{1-3}$, agricultural pests ${ }^{4,5}$, and invasive species ${ }^{6,7}$. Several different GE biocontrol strategies have been proposed, each with unique strengths and weaknesses ${ }^{8}$. Key differences exist in the mechanisms resulting in different degrees of scalability (e.g. as a result of egg versus adult release) and differences in the likeliness and fitness of resistance mutations.

Release of insects carrying dominant lethals (RIDL) is a proven technology that has been demonstrated in various insects ${ }^{9,10}$. In RIDL, a 'self-limiting' gene is controlled by a small-molecule repressible promoter. The self-limiting gene is one that reduces the fitness of the organisms upon expression, either by causing lethality or a deleterious phenotype. Propagation of the GE biocontrol agent in the presence of the small-molecule repressor keeps the self-limiting gene turned off. Hybrids between released RIDL adults and wild mates experience embryonic or lateonset lethality in the absence of the repressor due to the unregulated expression of the selflimiting gene.

Sex-ratio biasing GE biocontrol agents have been engineered via several mechanisms ${ }^{6,11}$. Female-lethal strains can be designed analogous to RIDL insects, but using a repressible femalespecific promoter or alternative splicing variant to control expression of the self-limiting gene. Alternatively, sex-ratio biasing can be engineered with $\mathrm{X}$-shredder designs that express nucleases to cleave the X-chromosome during meiosis, resulting in only male offspring ${ }^{12,13}$. In species whose sex determination is influenced by both genetics and environment, $Y Y$ females can be produced for an approach known as 'Trojan $Y$ chromosomes' ${ }^{\prime 4}$. Released $Y Y$ females have only male offspring and skew the sex ratio in subsequent generations.

The development of sequence-programmable nucleases, especially CRISPR/Cas9-based systems, has accelerated the construction of threshold-independent meiotic gene drives ${ }^{12,15,16}$. In meiotic gene drives, 'drive' alleles encode a sequence programmable nuclease expressed during meiosis ${ }^{17}$. Expression of the nuclease leads to cutting of a wild allele and replacement with the drive by gene conversion via homology-directed repair. Using promoters that direct expression of the drive allele early in gametogenesis, an organism heterozygous for the drive allele in somatic tissue will become homozygous in the germline, only passing on the drive allele to its offspring. 'Suppression gene drives' (SGDs) target a haplosufficient essential gene and will cause a population to crash once the drive allele has spread through the population ${ }^{12}$.

We have recently demonstrated a strategy for GE biocontrol called synthetic genetic incompatibility (SGI) ${ }^{18}$. SGI approximates the behavior of a single-locus extreme underdominance system, which have been noted for their theoretical applications in population control ${ }^{19}$. SGI is engineered by designing programmable transcription activators (PTAs) to drive lethal overexpression or ectopic expression of endogenous genes in hybrid embryos that are produced when GE organisms mate with wild-type. Thus, any mating event between and SGI organism and wild-type will fail to produce viable offspring ${ }^{18}$. A variation of SGI which specifically targets 
gametogenesis by limiting PTA expression to the germline would result in hybrids that are viable but sterile.

Wide-spread use of GE insects for population control is likely in the near future. Technologies that use RIDL for the control of agricultural pests ${ }^{4}$ and disease vectors ${ }^{20}$ have been approved for field trials in the US by the USDA, FDA, and EPA. Sex-ratio biasing constructs have been developed in several insect species as well as in vertebrate pests ${ }^{6,21-23}$. Meiotic gene drives have been constructed in laboratories and tested in caged studies to suppress populations of mosquitoes and fruit flies ${ }^{12,24,25}$. All of the components required to engineer an SGI strain have been shown to function properly in $D$. melanogaster ${ }^{26}$. Predicting the performance qualities of these population control strategies using computational models and simulations is an important prerequisite to laboratory or field trials.

Here we introduce a new GE biocontrol strategy, named Field-Amplified Male Sterility System (FAMSS) that combines sterilizing SGI with female-lethality. We assess the performance of FAMSS and alternative GE biocontrol agents using a spatially-defined agent-based simulation model of Aedes aegypti populations. As the primary vector of multiple viruses including Dengue, Zika, and Chikungunya, Ae. aegypti is a critical target for applications in disease control ${ }^{27}$. We show that FAMSS is a spatially and temporally self-limiting strategy to control Ae. aegypti populations. Lastly, we show that the FAMSS strategy outperforms suppression gene drives in the context of genetic resistance because of differences in relative fitness of resistant organisms.

\section{Results}

\section{Agent-based simulation model for Aedes aegypti.}

We developed an agent-based model for Aedes aegypti that allows us to track the genotype of every individual in an arbitrary number of neighboring populations (Figure 1 and Supplementary Note 1). The model is an adaptation of the simulation described by Dye ${ }^{28}$ and is based on empirical data from the 1966 World Health Organization Aedes Research Project at the half hectare Wat Samphaya in Bangkok, Thailand ${ }^{29}$. Whereas the Dye model treats adulthood as a single black-box, our model includes more detailed progression through multiple gonotrophic cycles (GCs). Because of this, we incorporate additional empirically-defined parameters on daily survivorship, egg deposition per GC, and gestation time ${ }^{30}$. Unlike more recent simulation models that track fine-scale movements of mosquitoes between specific habitat features such as standing water pools, and blood sources ${ }^{31,32}$, our model only considers spatial movements in terms of migration between neighboring populations. This last feature allows us to predict what will happen in border regions where GE mosquitoes can interact and crossbreed with non-targeted wild-type populations.

Within a single population, individual agents in our model progress through a series of 37 discrete states (life-stages) in time-steps of 1.57 days (Figure 1a,b). These states include larval, pupal, and adult stages. Adult females mate with a random male in the population during early adulthood. Sperm from this mating event is stored and used to fertilize eggs at the start of up to four gonotrophic cycles (GCs). However, the daily mortality rates are sufficiently high so that the average female will only go through one GC. For mating events that produce embryonic lethality, 
inviable embryos are 'killed' between life-stages 1 and 2 (hatching of eggs) (Figure 1a). Specific examples of mating events that result in embryonic lethality are described in subsequent sections.

A wild-type population of mosquitoes reaches equilibrium due do density-dependent mortality rates during $L 1$ and $L 2$ instars. We have tuned the larval density-dependent mortality rates within the range of measured values such that the steady state population size fits observations from the Bangkok study ${ }^{29}$ (Supplementary Note 2). In our model, equilibrium is reached at approximately 4000 total agents including 600 adults (Figure 1c). The number of adults estimated in the Bangkok study ranges between 460 and 2130 during different months ${ }^{29}$. Further, our simulation model shows expected behavior characteristics for Ae. aegypti populations including monotonic stability and robustness to changes in fecundity ${ }^{28}$. We have included a rough spatial component in our model that allows tracking of allele frequencies between neighboring populations. We include a parameter termed 'migration rate' that describes the likelihood that a given adult mosquito will migrate into a neighboring population at each time step (Figure 1d). Studies on the migration rates of individual mosquitoes in the modeled Wat Samphaya population show that movement is limited to only tens meters per day ${ }^{29}$, which coincides with the range of migration rates tested here. All of the parameters in our model can be changed to account for the unique population dynamics specific to a location of interest.

\section{Efficacy of genetic population control methods on individual populations.}

We performed 200 time step simulations for alternative GE population control methods, including female-lethality, SGI, and SGD (Figure 2a-c). For each strategy, we simulated a onetime release of GE mosquitoes at different starting frequencies of GE and wild-type. We performed each experiment in triplicate and report the mean population composition at each timestep as well as the standard deviation of the mean. Our results confirm the expected behavior of each modeled control strategy.

We model the FL system as the previously reported 'flightless female' Ae. aegypti ${ }^{33-35 .}$ Released GE mosquitoes are given the genotype 'LL' (wild-type are 'Il'), and the flightless phenotype is present in females with at least one ' $L$ ' allele. We simulate the flightless female phenotype by removing them from the model at the first adult life-stage. The FL strategy displayed a temporally self-limiting population suppression that scaled with initial release rate (Figure 2a). After initial release, the fraction of the population comprising wild-type individual eventually returned to $100 \%$ as the FL genotype was diluted out.

The SGI release strategy was modeled for population replacement applications where both male and female SGI mosquitoes are released. We encoded the SGI genotype as two independently segregating genetic loci. 'P' denotes a mutated promoter in a lethal overexpression gene target and ' $T$ ' denotes the presence of a programmable transcription activator 18,26. GE mosquitoes have the genotype 'PPTT', and wild-type have the genotype 'pptt'. Any embryo that contains at least one ' $T$ ' allele and at least one ' $p$ ' allele is inviable. With release of both sexes, SGI is expected to act as a threshold-dependent gene drive. At frequencies greater than $50 \%$, the SGI genotype is expected to drive to fixation. At frequencies less than $50 \%$, the wild-type genotype is expected to drive to fixation. We observe a clear threshold of $50 \%$ population composition in the simulation models (Figure $2 \mathrm{~b}$ ), but note that achieving fixation required an initial release of $70 \%$ SGI agents. The reason for the discrepancy has to do with differing life-stages of the initial populations of wild-type versus engineered individuals. In our 
simulations, the starting wild-type population contains a distribution of life stages (e.g. larval, pupal, adult) that represents a steady-state untreated population. The released bolus of GE organisms only contains individuals in the egg/larval stages. We do this to simulate realistic biocontrol applications. Because of density dependent mortality rates at larval stages, an initial release rate of $50 \%$ is quickly reduced to a sub-50\% population frequency (Figure $2 \mathrm{~b}$ ). It is noteworthy that even for some release rates that do not result in fixation of the SGI genotype, there is a substantial wild-type population suppression effect that lasts 50-100 time steps (Figure $2 b$, right).

We modeled SGD applications using a genotype similar to that previously developed suppressive meiotic drive in Ae. aegypti ${ }^{12,36}$. Released SGD mosquitoes have the genotype 'GW' (compared to 'WW' for wild-type mosquitoes), signifying that they are heterozygous for the drive allele in somatic tissue. For the purpose of the simulation in Figure 2c, we assumed ideal drive behavior (100\% homing rate, $0 \%$ non-homologous end joining repair) to show that this thresholdindependent gene drive functions as predicted in our simulation model. At release rates between $90 \%$ and $10 \%$, the SGD always drives to fixation. In all cases, the population of wild-type mosquitoes is completely suppressed with only the single initial release of GE biocontrol agents. (Figure 2c right).

\section{Combining genetic incompatibility with female lethality for genetically-encoded sterile male release.}

Next, we simulated a combination GE control method that entails engineering both FL and SGI into a single biocontrol strain. We named this approach Self-Sorting Incompatible Male System (SSIMS), because GE males and females can be released together to suppress a wild population. There are two feasible release strategies. First, the released individuals could be reared in the presence of tetracycline (to repress FL construct) and released as adults. This scenario would be ideal for pests or organisms that live and reproduce for many years (e.g. invasive carp), as SSIMS males and females will both suppress the population when they mate with wild-type. If they mate with each other, only SSIMS males are viable and will amplify the suppressive effect. The second scenario, more applicable for mosquito control, is to release SSIMS agents as eggs. Only males will survive developmental stages in the absence of tetracycline, and thus sex-sorting occurs in the field. The latter release scenario was simulated here.

We modeled SSIMS with three independently segregating genetic loci. With allele names representing the same molecular components described in the previous section, SSIMS agents have the combined genotype 'PPTTLL', and wild-type have the combined genotype 'ppttll'. As shown in Figure 2d, the SSIMS strategy is temporally self-limiting. As we simulate the release of eggs/larva there is no field amplification resulting from SSIMS males mating with SSIMS females. We observed population suppression that scaled with increased release rates. This approach is more strongly temporally self-limited than FL alone, as the genotype cannot be passed on to future generations (note difference of persistence between FL and SSIMS in Figures 2a and 2d).

To achieve moderate field amplification of the suppressive effect afforded by genetic incompatibility, we modeled a second combinatorial control strategy named Field-Amplified Male Sterility System (FAMSS). FAMSS is similar to SSIMS except that the programmable transcription activator is only expressed in germ-line tissue (Figure 2e). With this genetic design, hybrids from wild-type $x$ engineered matings would be viable but sterile ${ }^{37}$. This provides one extra generation 
of field-amplification compared to SSIMS. Our simulations (Figure 2e) reflect this amplification, with suppressive behavior that is stronger and more long-lasting than SSIMS. Compared to FL alone, FAMSS provides a stronger suppressive effect (Supplementary Figure S2) and is more stringently temporally self-limiting, with abrupt removal of the FAMSS genotype after approximately fifty time steps (Figure $2 \mathrm{e}$ ).

\section{Robustness of FAMSS and SGD to genetic resistance.}

Because the FAMSS strategy is self-limiting, a more realistic population suppression program would involve an initial bolus release of FAMSS individuals, followed by periodic small scale releases. These periodic releases will keep the population suppressed even in the event that new wild-type mosquitoes immigrate into the treated region. We simulated such periodic release programs for FL, SGD, SSIMS, and FAMMS with varying release numbers and frequencies (Supplementary Note 3). SGD was able to completely eradicate the wild-type population in every periodic release schedule. While this represents an unconventional and aggressive strategy for SGD release, we simulate it here for the sake of comparison and note that the decreased time to eradication will result in improved robustness to genetic resistance. Of the remaining strategies, FAMSS was the most powerful and was able to locally eradicate the wild-type population with an initial bolus release of $90 \%$ followed by weekly release of 800 eggs/larva.

Next, we modeled the stochastic emergence of genetic resistance for each control strategy (Figure 3). For SGDs to function ideally, all nuclease-induced double-strand DNA breaks would be repaired by homology-directed repair, using the drive allele as a repair template. However, if the double-strand DNA break repairs by non-homologous end joining (NHEJ) or microhomology mediated end joining (MMEJ), the repaired locus can contain mutations that confer resistance to future cutting (Figure $3 a)^{25,36}$. We model this as the conversion of a wild-type allele 'W' to a resistant allele ' $R$ '. It is also possible that the NHEJ repair will create a non-functional allele, which we designate 'I'.

Empirical measurements of resistance mutations in laboratory populations of Ae. aegypti and $D$. melanogaster point to complex and multifactorial rates of resistance that arise from differences in NHEJ repair frequency in different cell types ${ }^{25,36}$. To simplify this process in our model, we assume resistance arise only from NHEJ during gametogenesis. Keeping the homing frequency fixed at $98 \%$, we modeled rates of NHEJ from $0 \%$ to $5 \%$. In our model, an NHEJ rate of $2-2.5 \%$ closely approximates the experimentally observed behavior, with SGD alleles increasing in the population for approximately five generations before dissipating due to selection for resistant alleles (Supplementary Note 4).

Genetic resistance to SGI (and by extension to SSIMS or FAMSS) could emerge from silencing of the PTA, sequence diversity in the region targeted by the PTA, and by promoter conversion in the hybrid embryo. The first two mechanisms of resistance can be buffered by engineering strategies ${ }^{18}$ and were not observed frequently in a model yeast system. Promoter conversion occurs when the engineered resistant promoter of the SGI parent replaces the susceptible wildtype promoter in the hybrid embryo (Figure 3b). This form of resistance occurred at a frequency of approximately $10^{-3}$ in yeast ${ }^{18}$. In our model, we simulate resistance by promoter conversion using a by allowing offspring of FAMSS and wild-type parents ('PPTTLL' and 'ppttll', respectively) to have a viable genotype of 'PPTtLl' at a rate defined by the promoter conversion frequency parameter. 
To determine how the FAMSS and SGD strategies perform when challenged with genetic resistance, we ran models simulating a periodic release of biocontrol agents with frequencies of promoter conversion or NHEJ, respectively, spanning three orders of magnitude (Figure 3c/d). For each rate of NHEJ tested, resistance emerged and drove down the frequency of SGD agents in the simulation (Figure 3c). Complete population suppression occurred before the emergence of resistance only at NHEJ rates of $10^{-4}$ or less, in agreement with mathematical modeling of a population this size $^{38}$.

In stark contrast to the behavior of SGD, the FAMSS approach was robust to the emergence of resistance by promoter conversion (Figure 3d). Even with rates of promoter conversion of 0.1 (e.g. one in ten of the progeny between FAMSS and wild-type survive with a genotype of 'PPTtLl'), the FAMSS genotype quickly went to fixation and the dynamics of population suppression mirrored the simulation in the absence of resistance. This can be explained by examining the fitness of resistant individuals in relation to wild-type or FAMSS (Figure 3e). The 'PPTtLl' escapee genotype is less fit than either the wild-type or FAMSS genotype. Half of the offspring between an escapee and a wild-type would be inviable as a result of inheriting a programmable transcription activator ' $T$ ' and susceptible promoter ' $p$ '. Half of the offspring between an escapee and a FAMSS mosquito would effectively regenerate the FAMSS genotype. Because the wildtype genotype continues to be suppressed in the presence of escapees, the periodic addition of more FAMSS mosquitoes gradually dilutes out the escapee genotype, leading to robust population suppression (Figure 3d).

\section{Behavior of FAMSS and SGD for spatially-defined population control.}

The ability of FAMSS or SGD strategies to rapidly suppress a population of wild-type Ae. aegypti would be counteracted in real-life applications by wild-type individuals immigrating into the treatment area. To examine this scenario, we performed a spatially-explicit population suppression experiment by simulating 49 individual populations on a $7 \times 7$ grid. All 49 cells were seeded with a steady-state starting population of wild-type mosquitoes (approximately 4000 individuals). A $3 \times 3$ grid in the center was treated with GE population suppression agents (Figure 4a). Each sub-population was monitored across 200 time steps (Figures 4b-d). At each time step, adult mosquitoes could stochastically emigrate out of their current cell and into one of eight neighboring cells (diagonal moves are permitted). The $7 \times 7$ grid was simulated on a toroid landscape, so agents in the top row could emigrate 'up' out of their cell and into the bottom row. We simulated the same release schedule as described above (90\% initial release followed by 800 individuals every 5 time steps), with a migration rate of 0.01 for both SGD (Figure 4c) and FAMSS (Figure 4d) strategies.

Two important observations were made from the spatially-explicit treatment model using SGD agents (Figure 4c). First, the SGD genotype did not remain confined to the treatment cells. SGD individuals quickly spread throughout the $7 \times 7$ grid, where they transiently suppressed untreated Ae. aegypti populations. Second, a wave of resistant organisms shortly followed and also spread throughout the grid. This result is not surprising and has been shown by others employing different modeling techniques ${ }^{38,39}$. Second, SGD is less robust to emergence of genetic resistance in the multi-population simulation compared to the single population simulations. In single populations models, rates of NHEJ of $10^{-3}$ were sufficiently low to prevent the emergence of resistance in two of three replicates (Figure $3 \mathrm{c}$ ) and rates of $10^{-4}$ saw no resistant individuals emerge (data not 
shown). In contrast to this, we consistently see resistant organisms emerge in the multi-population simulations with NHEJ rates of $10^{-4}$, signifying that SGD is less robust to challenges by genetic resistance in the multi-population simulations. Each mating event en route to full eradication is an opportunity for resistance to evolve and spread, and more mating events are required for suppression in the multi-population simulation. Importantly, this is not just function of increasing total population size ${ }^{38}$, but the rate of migration between sub-populations.

In contrast to SGD, the FAMSS approach maintained a spatially-limited zone of population suppression throughout the experiment (Figure 4d), confined to the $3 \times 3$ grid into which the biocontrol agents are released. Also, the robustness to genetic resistance does not change as a function of population size or structure. As is the single-population simulations, the frequency of resistance mutants in the population does not grow to fixation, even at relatively high rates of promoter conversion.

\section{Discussion}

Our agent-based simulation model reproduces the overall behavior of several GE biocontrol strategies to suppress or replace populations of the important disease vector, Ae. aegypti. Embedded in the model are genetic rules that allow us to simulate female lethality, suppression gene drives, synthetic genetic incompatibility, and combination treatments. We have focused our experiments in this study to compare FAMSS with SGD in the context of genetic resistance. Performance differences between FAMSS and RIDL or Sex-ratio biasing do exist, but are more strongly impacted by parameters like fitness or mating competitiveness. Meaningful comparisons between these approaches will require empirical measurements of these parameters in engineered insects ${ }^{35}$.

We have incorporated into our models the likely mechanisms of genetic resistance for both FAMSS and SGD approaches. Resistance to SGD is well-documented in experimental systems 25,36 and has tempered the enthusiasm of using meiotic gene drives for population suppression ${ }^{40}$. Several approaches to slow the evolution of resistance have been proposed, such as employing multiple guide $\mathrm{RNAs}^{38}$ or manipulating the ratio of homology directed repair to NHEJ ${ }^{41}$. As the field makes progress towards overcoming resistance to SGDs, it is important to consider the performance in spatially-explicit applications. We show here that a latent population of wild-type organisms that can slowly migrate into a treatment area will exacerbate the problem of resistance, and require that rates of resistance are several orders of magnitude lower than what would be sufficient to suppress an isolated population.

Our proposed FAMSS strategy fares well when challenged with genetic resistance, both in isolated populations and in spatially-explicit models. The engineered SGI genetic construct essentially behaves as a single locus underdominant Dobzhansky-Muller Incompatibility (DMI) ${ }^{42-}$ ${ }^{44}$, which act as stable species barriers to gene flow in neighboring populations ${ }^{45}$. The population genetics of DMls are thought to be the driving force behind natural speciation, and the sheer number of species that have existed on our planet speaks to the difficulty in overcoming these barriers once they exist ${ }^{43}$. The FAMSS strategy we describe here, and the SGI approach in general, is robust to high frequencies of genetic resistance for the same reason.

We considered only a single mechanism of resistance, namely promoter conversion, although other mechanisms are possible. We have omitted other mechanisms of resistance because these can be addressed with simple engineering solutions. For example, silencing or mutation of the 
PTA can be circumvented by creating a positive selection module using an essential gene ${ }^{18}$. Resistance caused by underlying sequence diversity in the population would have a similar fate as promoter conversion mutants. The surviving hybrids will still one copy of the PTA and will be less fit than wild-type in subsequent mating events. Further, if two mutually incompatible FAMSS strains are engineered that drive overexpression of different target genes, these could be released in an alternating fashion. Genetic escapees from the first treatment will receive half of their genetic material from the first FAMSS strain, and can be suppressed with the second FAMSS strain. Unanticipated mechanisms of resistance will be learned from characterizing SGI insects, which are currently underway ${ }^{26}$.

Our spatially explicit model demonstrates the potential for FAMSS to provide long-term suppression of local mosquito populations even when challenged with genetic resistance. The migration rate used in our models ( $1 \%$ chance of migration per adult per time step) is reasonable given the recapture rates of mosquitoes in the field studies on which this model is based ${ }^{29}$. If the migration rates in a specific geographic region are greater or less that those modeled here, it will affect the numbers of mosquitoes that need to be released, but will affect the overall performance of the biocontrol strategy. The same is true of relative fitness and mating competitiveness of FAMSS versus wild-type males. As empirical measurements of these variables become available for FAMSS mosquitoes, we will update our model to improve its accuracy.

One intriguing possibility is to use FAMSS or SGI mosquitoes as a safeguard to limit the spread of a SGD release ${ }^{46}$. If the two genotypes were incompatible (i.e. the SGD strain is has a wild-type promoter at the FAMSS target locus), then a population of FAMSS mosquitoes could be prereleased to form a 'firewall' that will limit the spread of the SGD. For example, a resident populations of FAMSS or SGI mosquitoes could be maintained around a port-of-call with SGD released in interior of an island. By decreasing the effective population size through which an SGD could spread, FAMSS could improve the practical efficacy of SGD or other thresholdindependent gene drives. Similarly, release of a large bolus of FAMSS agents could function as a fire blanket to immediately suppress an accidental or nefarious release of SGDs organisms.

In conclusion, we have described and simulated a new population control strategy that hardwires genetic incompatibility and sex-sorting into the genotype of a GE biocontrol strain. We show that this strategy is robust when challenged by high rates of genetic resistance in stark contrast to CRISPR/Cas9-based meiotic gene drives.

\section{Methods}

Agent-based simulation model. Our agent-based simulation model was written in Python ${ }^{47}$ on the Mesa platform ${ }^{48}$. Model parameters (of which select parameters are listed in Figure 1B) including density dependent and density independent survival rates for each life-stage, sex-ratio of new eggs, number of eggs per gonotrophic cycle, number of time steps for each life-stage, and initial number of wild-type agents were not varied during population control experiments. Variable parameters defined prior to each simulation include starting number of GE organisms, genotype of GE organisms, frequency of homing and NHEJ (SGD simulations only), frequency of promoter conversion (FAMSS/SSIMS/SGI simulations only), total number of time steps, number of GE organisms released at periodic time steps, number of time steps between periodic release, and 
migration rate. Agents added to the model at time step 0 are seeded at random life-stages, with $95 \%$ at life=stage 10 (last larval stage) or earlier and 5\% at adult stages. Annotated Python scripts for single population simulations and $7 \times 7$ grid simulations as well as README instruction files are available on GitHub.

Rules for behavior of GE control strategies. The genotype of each agent in the simulation is stored as the attribute self.genotype. To model FL, SGI, or FAMSS, each agent is given a six letter genotype, with two 'promoter' alleles ( $P$ or $p$ ), two PTA alleles ( $T$ or $t$ ), and two female lethality alleles ( $\mathrm{L}$ or $\mathrm{I})$. In each case, the lowercase allele designates wild-type. During the reproduction step, the genotype of each new egg produced is determined by a stochastic selection of alleles from each parent. Unless otherwise noted, each allele has a $50 \%$ chance of inheritance. Sex is determined stochastically with a 50:50 male:female sex ratio. All eggs are placed into the model scheduler and grid regardless of genotype.

To model SGD, we use a simplified two letter genotype for each agent in the model. Allele names all correspond to a single meiotic drive locus and can designate a gene drive (G), wild-type (W), resistance mutation $(\mathrm{R})$, or recessive lethal mutation (I). The $\mathrm{R}$ and I genotypes only arise when modeling NHEJ (discussed below).

Genotype viability is assessed during the progression from lifestage 1 (egg) to lifestage 2 (first instar larva). Individuals carrying a PTA (i.e. a T allele) AND a wild-type target promoter (i.e. a p allele) are inviable and are removed from the model. Females carrying the female-lethal construct (i.e. a L allele) are inviable and are removed from the model. For SGD populations, any organism lacking at least one $\mathrm{W}$ allele or $\mathrm{R}$ allele is inviable.

Genetic resistance in simulation model. We model promoter conversion events in SGI or FAMSS populations during the reproduction step. In any new egg that would be heterozygous at the promoter location (i.e. Pp or pP), the wild-type ' $p$ ' allele is changed to the resistant ' $q$ ' allele stochastically at a promoter conversion frequency specified at the start of the simulation. Because the rules for embryonic lethality require the co-existence of ' $T$ ' and ' $p$ ' alleles, these ' $q$ ' mutants remain viable. Functionally, this is equivalent to replacing the ' $p$ ' for an engineered ' $P$ ', but the ' $q$ ' designation facilitates future tracking of the mutated allele.

We model resistance in the SGD simulations at the stage of gametogenesis. For gene drive carriers ('WG' or 'GW'), the 'G' allele is passed on at a frequency of:

$$
F_{G}=0.5+0.5\left(F_{\text {homing }}\left(1-F_{N H E J}\right)\right)
$$

where $F_{\text {homing }}$ is the homing frequency and $F_{N H E J}$ is the frequency of non-homologous end joining. Best case gene drive simulations use a homing frequency of $100 \%$ and a non-homologous end joining frequency of $0 \%$. The gamete will inherit the $\mathrm{G}$ allele from a heterozygous parent stochastically if a random number between 0 and 1 is less than or equal to $F_{G}$.

Our model simulates non-homologous end joining if the random number generated, $\mathrm{N}$, lies in the following range:

$$
0.5+0.5\left(F_{\text {homing }}\left(1-F_{\text {NHEJ }}\right)\right)<N<0.5+0.5\left(F_{\text {homing }}\right)
$$

In this case, a resistant allele ' $R$ ' is passed on one third of the time and a haploinsufficient allele 'I' is passed on two thirds of the time. These probabilities are based on the random likelihood that an indel generated by NHEJ will result in an in-frame coding DNA sequence (CDS). Our model 
bioRxiv preprint doi: https://doi.org/10.1101/316406; this version posted May 7, 2018. The copyright holder for this preprint (which was not certified by peer review) is the author/funder. All rights reserved. No reuse allowed without permission.

assumes that any NHEJ repair that generates an in-frame CDS will still be haplosufficient and will resist further cutting by the drive nuclease. This is likely a slight overestimate of resistance given a particular NHEJ frequency, but is reasonable for the purposes of modeling. 


\section{References}

1. Alphey, L. et al. Genetic control of Aedes mosquitoes. Pathog. Glob. Health 107, 170179 (2013).

2. Harris, A. F. et al. Successful suppression of a field mosquito population by sustained release of engineered male mosquitoes. Nature Biotechnology 30, 828-830 (2012).

3. Alphey, L. et al. Malaria control with genetically manipulated insect vectors. Science 298, 119-121 (2002).

4. Harvey-Samuel, T. et al. Pest control and resistance management through release of insects carrying a male-selecting transgene. BMC Biol. 13, (2015).

5. Jin, L. et al. Engineered female-specific lethality for control of pest lepidoptera. ACS Synth. Biol. 2, 160-166 (2013).

6. Thresher, R. et al. Sex-ratio-biasing constructs for the control of invasive lower vertebrates. Nat. Biotechnol. 32, 424-427 (2014).

7. Thresher, R. E. et al. Genetic control of invasive fish: Technological options and its role in integrated pest management. Biol. Invasions 16, 1201-1216 (2014).

8. Alphey, L. Genetic Control of Mosquitoes. Annu. Rev. Entomol. 59, 205-224 (2014).

9. Thomas, D. D., Donnelly, C. A., Wood, R. J. \& Alphey, L. S. Insect population control using a dominant, repressible, lethal genetic system. Science (80-. ). 287, 2474-2476 (2000).

10. Harris, A. F. et al. Field performance of engineered male mosquitoes. Nat. Biotechnol. 29, 1034-1037 (2011).

11. Heinrich, J. C. \& Scott, M. J. A repressible female-specific lethal genetic system for making transgenic insect strains suitable for a sterile-release program. Proc. Natl. Acad. Sci. U. S. A. 97, 8229-8232 (2000).

12. Hammond, A. et al. A CRISPR-Cas9 gene drive system targeting female reproduction in the malaria mosquito vector Anopheles gambiae. Nat. Biotechnol. 1-8 (2015).

doi: $10.1038 /$ nbt.3439

13. Galizi, R. et al. A synthetic sex ratio distortion system for the control of the human malaria mosquito. Nat. Commun. 5, (2014).

14. Gutierrez, J. B. \& Teem, J. L. A model describing the effect of sex-reversed $Y Y$ fish in an established wild population: The use of a Trojan $Y$ chromosome to cause extinction of an introduced exotic species. J. Theor. Biol. 241, 333-341 (2006).

15. Esvelt, K. M., Smidler, A. L., Catteruccia, F. \& Church, G. M. Concerning RNA-guided gene drives for the alteration of wild populations. Elife 3, e03401 (2014).

16. Gantz, V. . \& Bier, E. The mutagenic chain reaction: a method for converting heterozygous to homozygous mutations. Science (80-. ). DOI:10.112, 1-2 (2015).

17. Sinkins, S. P. \& Gould, F. Gene drive systems for insect disease vectors. Nat. Rev. Genet. 7, 427-435 (2006).

18. Maselko, M., Heinsch, S. C., Chacón, J., Harcombe, W. \& Smanski, M. J. Engineering 
species-like barriers to sexual reproduction. Nat. Commun. 8, (2017).

19. Davis, S., Bax, N. \& Grewe, P. Engineered Underdominance Allows Efficient and Economical Introgression of Traits into Pest Populations. J. Theor. Biol. 212, 83-98 (2001).

20. Harris, A. F. et al. Field performance of engineered male mosquitoes. Nat. Biotechnol. 29, 1034-1037 (2011).

21. Yan, Y., Linger, R. J. \& Scott, M. J. Building early-larval sexing systems for genetic control of the Australian sheep blow fly Lucilia cuprina using two constitutive promoters. Sci. Rep. 7, (2017).

22. Concha, C. et al. A transgenic male-only strain of the New World screwworm for an improved control program using the sterile insect technique. BMC Biol. 14, (2016).

23. Li, F. \& Scott, M. J. CRISPR/Cas9-mediated mutagenesis of the white and Sex lethal loci in the invasive pest, Drosophila suzukii. Biochem. Biophys. Res. Commun. 469, 911-916 (2016).

24. Gantz, V. M. et al. Highly efficient Cas9-mediated gene drive for population modification of the malaria vector mosquito Anopheles stephensi. Proc. Natl. Acad. Sci. 201521077 (2015). doi:10.1073/pnas.1521077112

25. Champer, J. et al. Novel CRISPR/Cas9 gene drive constructs in Drosophila reveal insights into mechanisms of resistance allele formation and drive efficiency in genetically diverse populations. PLoS Genet. 13, e1006796 (2017).

26. Waters, A. J. et al. Rationally-engineered reproductive barriers using CRISPR \& CRISPRa: an evaluation of the synthetic species concept in Drosophila melanogaster. bioRxiv (2018). doi:10.1101/259010

27. Kraemer, M. U. G. et al. The global distribution of the arbovirus vectors Aedes aegypti and Ae. Albopictus. Elife 4, (2015).

28. Dye, C. Models for the Population Dynamics of the Yellow Fever Mosquito, Aedes aegypti. J. Anim. Ecol. 53, 247-268 (1984).

29. Sheppard, P. M., Macdonald, W. W., Tonn, R. J. \& Grab, B. The Dynamics of an Adult Population of Aedes aegypti in Relation to Dengue Haemorrhagic Fever in Bangkok. J. Anim. Ecol. 38, 661-702 (1969).

30. Goindin, D., Delannay, C., Ramdini, C., Gustave, J. \& Fouque, F. Parity and longevity of aedes aegypti according to temperatures in controlled conditions and consequences on dengue transmission risks. PLoS One 10, 1-21 (2015).

31. Magori, K. et al. Skeeter Buster: A stochastic, spatially explicit modeling tool for studying Aedes aegypti population replacement and population suppression strategies. PLoS Negl. Trop. Dis. 3, (2009).

32. Legros, M. et al. Evaluation of Location-Specific predictions by a detailed simulation model of Aedes aegypti populations. PLoS One 6, (2011).

33. Fu, G. et al. Female-specific flightless phenotype for mosquito control. Proc. Natl. Acad. Sci. 107, 4550-4554 (2010).

34. Wise de Valdez, M. R. et al. Genetic elimination of dengue vector mosquitoes. Proc. Natl. 
Acad. Sci. 108, 4772-4775 (2011).

35. Facchinelli, L. et al. Field Cage Studies and Progressive Evaluation of GeneticallyEngineered Mosquitoes. PLoS Negl. Trop. Dis. 7, (2013).

36. Hammond, A. M. et al. The creation and selection of mutations resistant to a gene drive over multiple generations in the malaria mosquito. PLoS Genet. 13, 1-16 (2017).

37. Thailayil, J., Magnusson, K., Godfray, H. C. J., Crisanti, A. \& Catteruccia, F. Spermless males elicit large-scale female responses to mating in the malaria mosquito Anopheles gambiae. Proc. Natl. Acad. Sci. 108, 13677-13681 (2011).

38. Marshall, J. M., Buchman, A., Sánchez, C. H. M. \& Akbari, O. S. Overcoming evolved resistance to population-suppressing homing-based gene drives. Sci. Rep. 7, 1-12 (2017).

39. Noble, C., Adlam, B., Church, G. M., Esvelt, K. M. \& Nowak, M. A. Current CRISPR gene drive systems are likely to be highly invasive in wild populations. bioRxiv 219022 (2017). doi:10.1101/219022

40. Bull, J. J. \& Malik, H. S. The gene drive bubble: New realities. PLoS Genet. 13, 2-5 (2017).

41. Champer, J., Buchman, A. \& Akbari, O. S. Cheating evolution: Engineering gene drives to manipulate the fate of wild populations. Nature Reviews Genetics 17, 146-159 (2016).

42. Dobzhansky, T. Studies on hybrid sterility - I. Spermatogenesis in pure and hybrid Drosophila pseudoobscura. Zeitschrift für Zellforsch. und Mikroskopische Anat. 21, 169223 (1934).

43. Muller, H. J. Isolating mechanisms, evolution and temperature. Biol. Symp 6, 71-125 ST - Isolating mechanisms, evolution and t (1942).

44. Presgraves, D. C. The molecular evolutionary basis of species formation. Nature Reviews Genetics 11, 175-180 (2010).

45. Bank, C., Bürger, R. \& Hermisson, J. The limits to parapatric speciation: DobzhanskyMuller incompatibilities in a continent-Island model. Genetics 191, 845-863 (2012).

46. Akbari, O. S. et al. Safeguarding gene drive experiments in the laboratory. Science (80). 349, 927-929 (2015).

47. Van Rossum, G. \& Et Al. The Python programming language. Python Softw. Found. (2010). at <http://www.python.org>

48. Mesa. at <http://mesa.readthedocs.io/en/latest/overview.html> 
Figure 1

a.

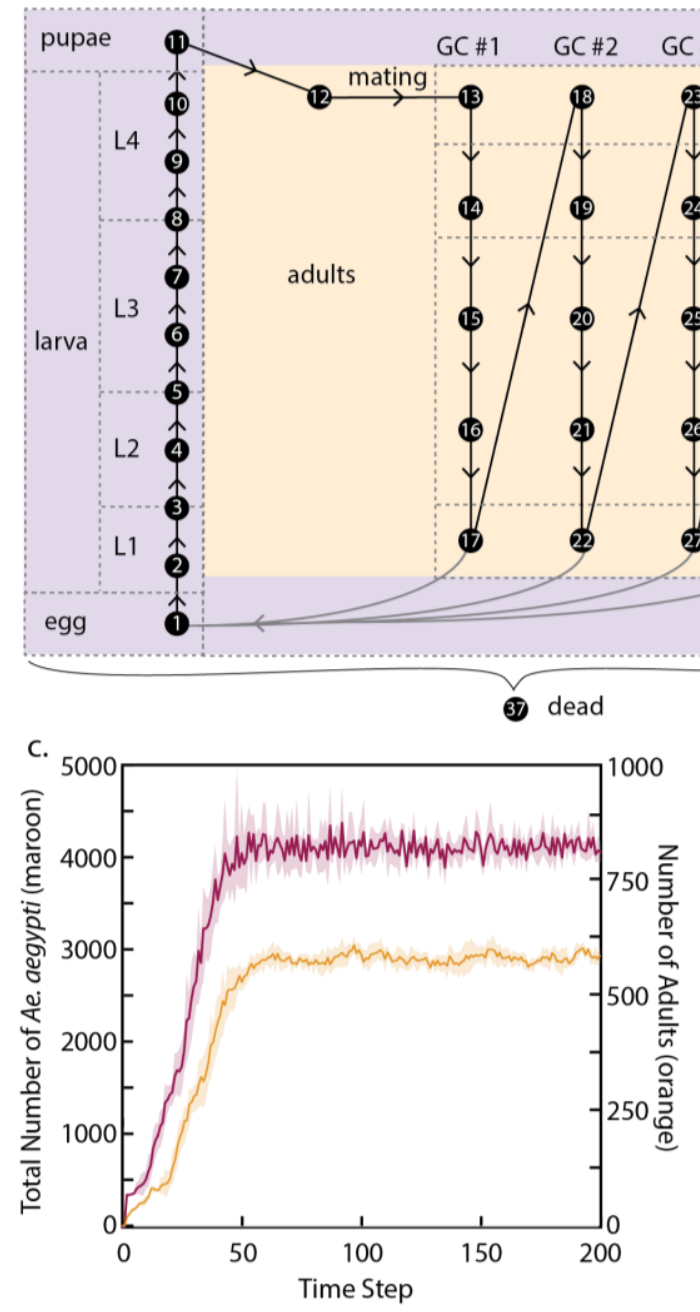

b.

Key Model Parameters

\begin{tabular}{ccc}
\hline Parameter & Value & Reference \\
\hline Eggs per GC & 40 & Goindin 2015 \\
Sex ratio & $50: 50$ & Dye 1984 \\
$\begin{array}{c}\text { L1 density-dependent } \\
\text { mortality }\end{array}$ & $0.0005 E^{0.91}$ & Sheppard 1969 \\
$\begin{array}{c}\text { L2 density-dependent } \\
\text { mortality }\end{array}$ & $0.0007 L^{0.8}$ & Sheppard 1969 \\
$\begin{array}{c}\text { 3 density-independent } \\
\text { mortality }\end{array}$ & 0.2 & Dye 1984 \\
$\begin{array}{c}\text { 4 density-independent } \\
\text { mortality }\end{array}$ & 0.2 & Dye 1984 \\
$\begin{array}{c}\text { Eclosion likelihood } \\
\begin{array}{c}\text { Adult daily survival } \\
\text { (male/female) }\end{array}\end{array}$ & $0.80 / 0.92$ & Sheppard 1969 \\
\hline
\end{tabular}

Total Number of Ae. aegypti

d.

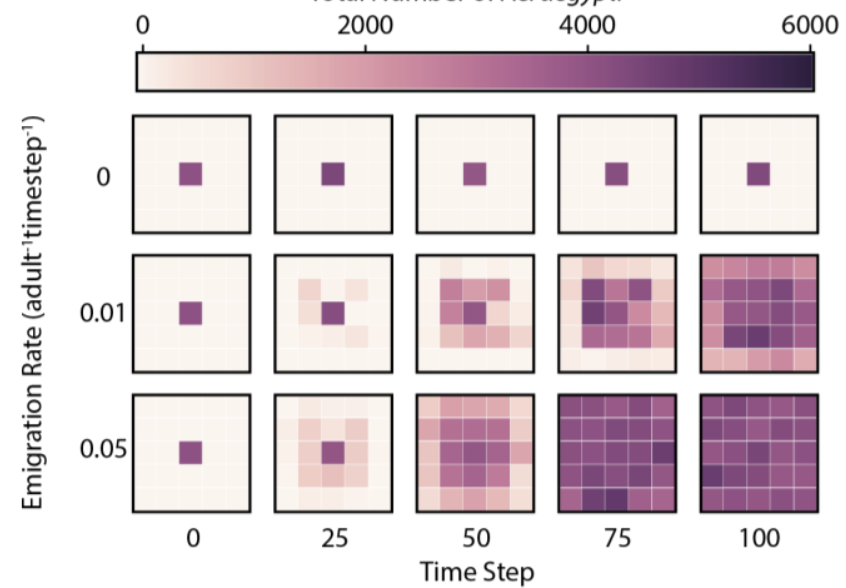

Figure 1. Agent-based simulation model for Aedes aegypti. (a) Schematic diagram of agentbased model, showing all possible life-stages (black nodes) for female mosquitoes. GC = gonotrophic cycle; L1-4 = larval instar stages I-IV. (b) List of key model parameters. (c) Results from simulation of a single population with initial size of 500 individuals (randomly distributed across life stages). Maroon trace shows mean number of agents with lifestage between 2 and 36 . Shadow shows standard deviation from five independent replicates. Orange trace shows mean number of adults with shadow denoting standard deviation from five independent replicates. (d) Migration between neighboring populations as a function of time. Results from three experiments, each run in triplicate, showing migration of $A$ e. aegypti from a seed population (center square on grid) to surrounding populations. Square color represents total number of individuals (lifestage 236) in each population at the time step labeled below. 
Figure 2

a.

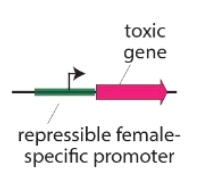

b.

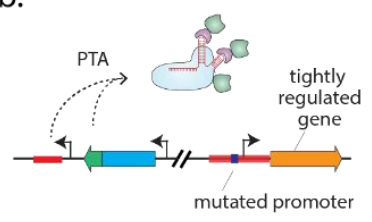

c.

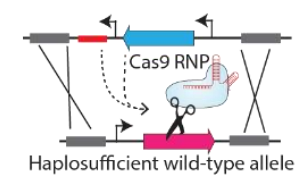

d.

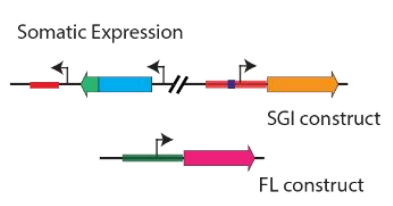

e.

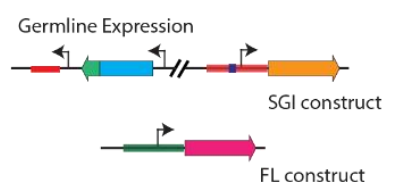

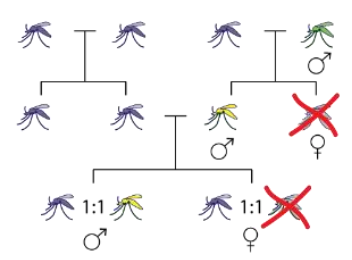
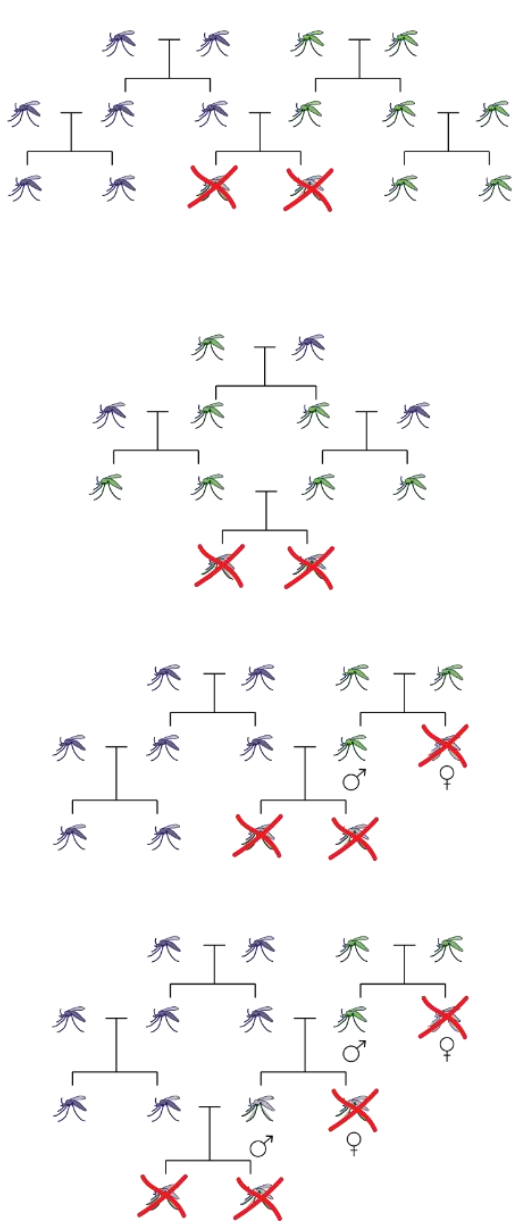
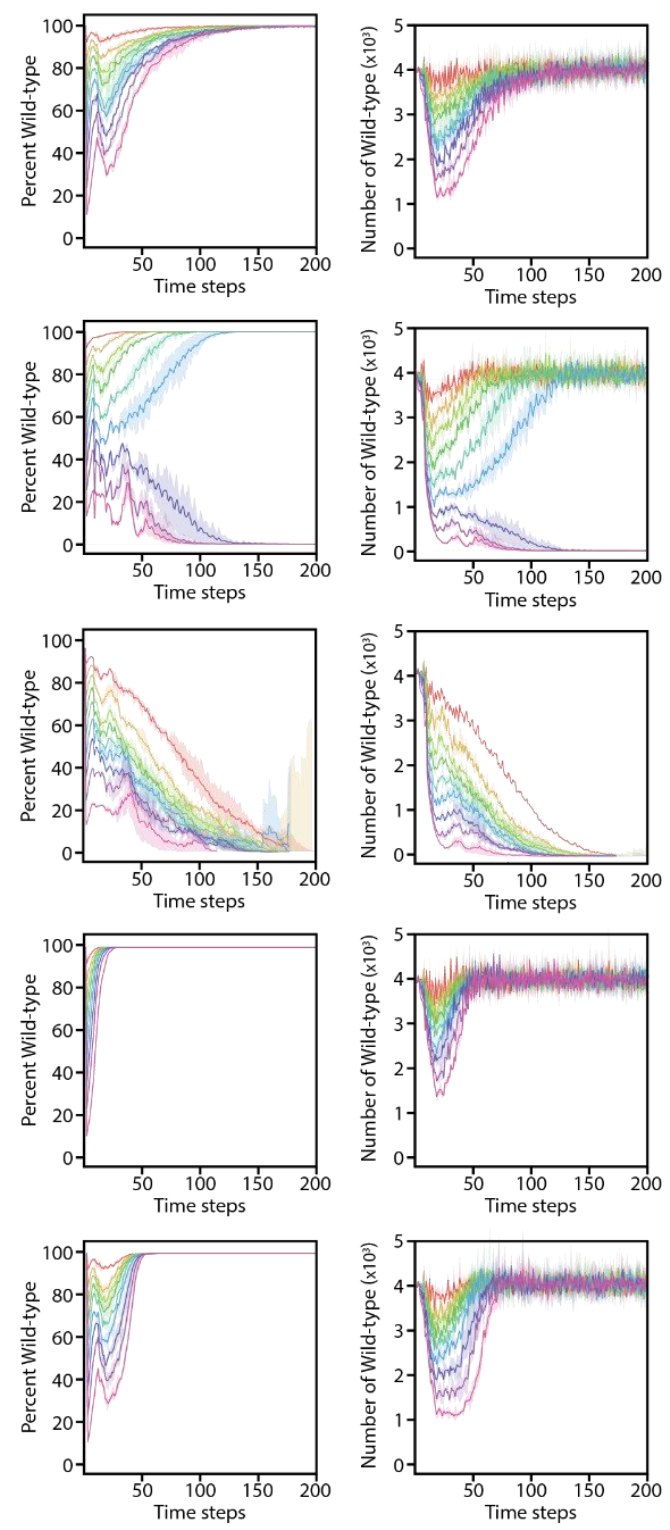

Figure 2. Comparison of alternative genetic population control methods in one-time release. Schematic drawing of genotype (left), mating outcomes of wild-type (purple) and GE (green) mosquitoes (center), and modeled performance at different release rates (right) for (a) flightless female (FL), (b) synthetic genetic incompatibility (SGI), (c) homing suppression gene drives (SGD), (d) self-stocking incompatible males (SSIMS), and (e) field amplified male sterility system (FAMSS). Performance graphs show the fraction simulated population comprised of GE mosquitoes (left plot) and the total number of wild-type mosquitoes in the simulation (right plot). Each simulation starts with steady-state (4000) wild-type agents and enough GE agents to comprise 10\% (red), 20\% (orange), 30\% (light green), 40\% (dark green), 50\% (light blue), 60\% (dark blue), $70 \%$ (indigo), $80 \%$ (violet), or $90 \%$ (magenta) of the starting population. Lines correspond to mean from three independent experiments, while back-shading denotes standard deviation. PTA, programmable transcription activator; RNP, ribonucleoprotein. 


\section{Figure 3}

a.
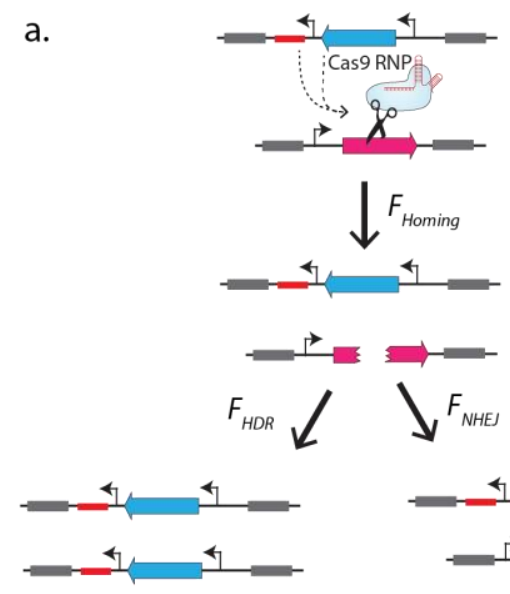

c.

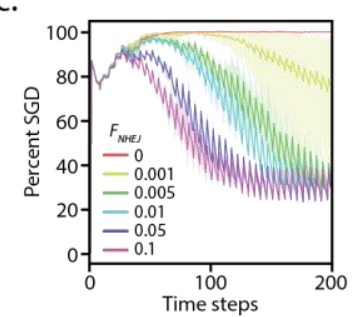

d.

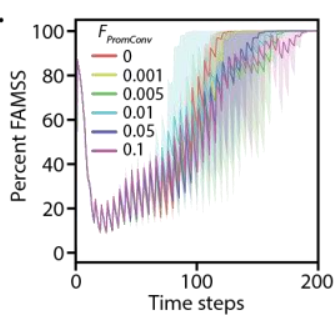

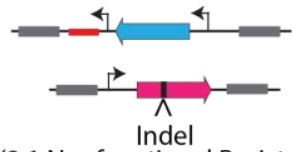

(2:1 Nonfunctional:Resistant) b.
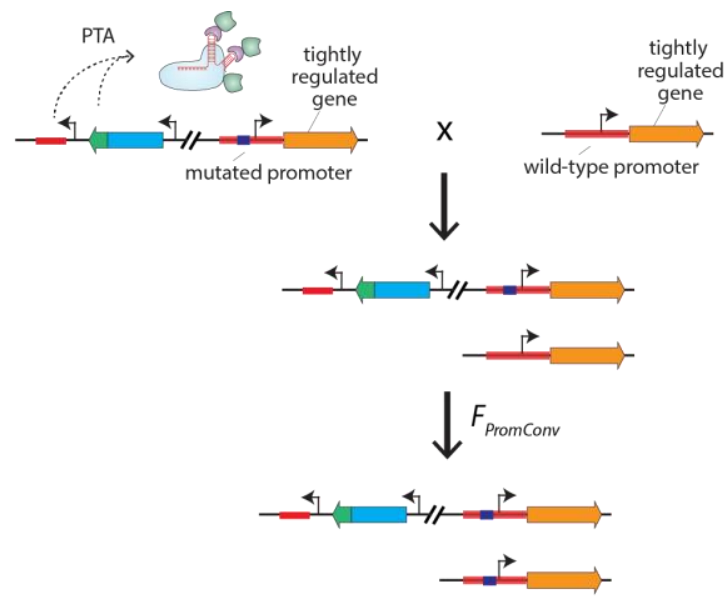

Figure 3. Performance of SGD and FAMSS in the context of genetic resistance. Mechanisms of simulated genetic resistance for SGD (a) and FAMSS (b) with key model parameters annotated. Performance of SGD (c) and FAMSS (d) with initial release rates of $90 \%$ GE mosquitoes and $10 \%$ wild-type, followed by release of $800 \mathrm{GE}$ larvae every 5 time steps. Plots show the percentage of the total population comprising mosquitoes with the GE biocontrol genotype (left), genetically resistant mosquitoes (center), and the number of adult females with either wild-type or resistant genotypes (right). Frequency of NHEJ or promoter conversion are given in the inset of the left plot. (e) Punnett square of possible mating events between promoter conversion escapee genotype and wild-type or FAMSS mating partners. 


\section{Figure 4}

a. 49 Population Simulation

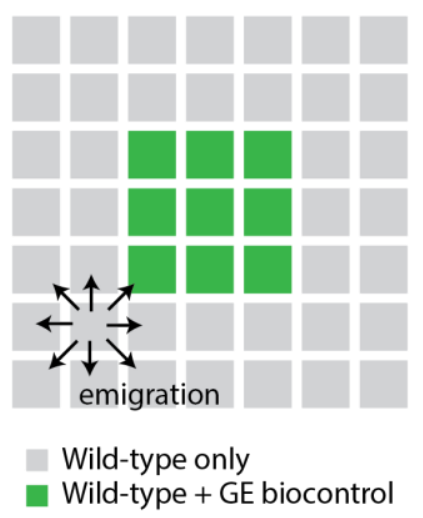

b.

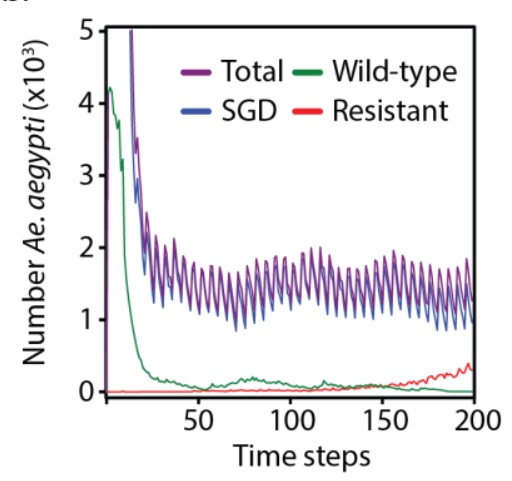

C.

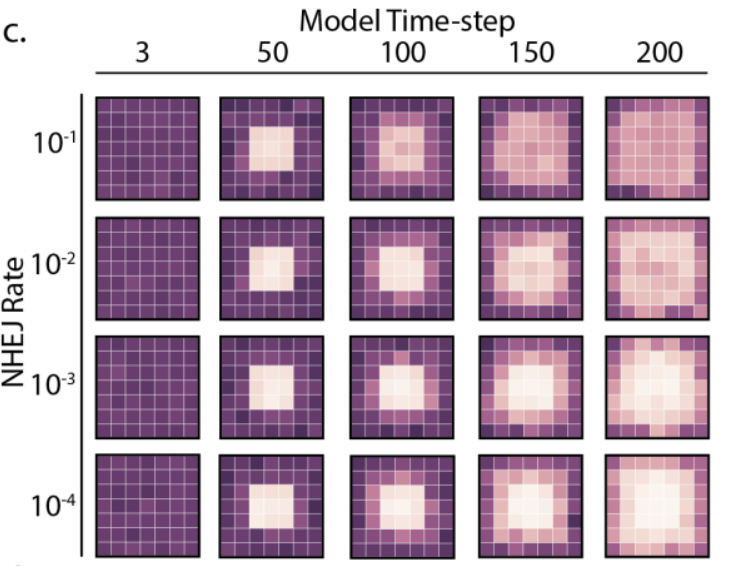

d.

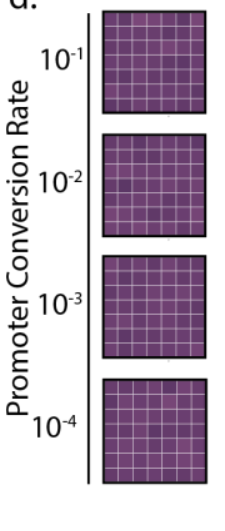

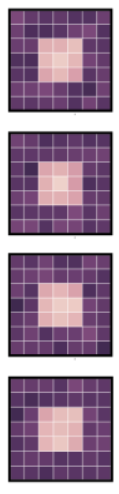

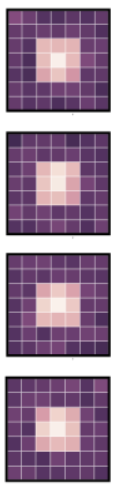

200
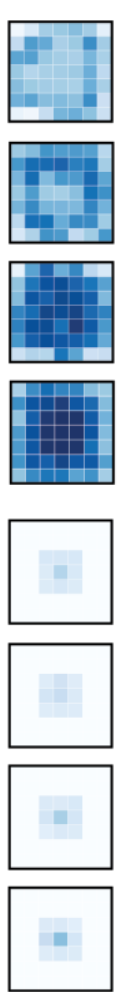

050100

Percent GE
200
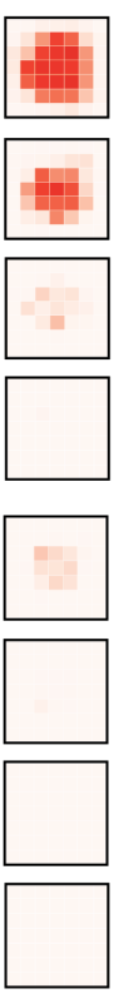

$0 \quad 50100$ Percent Resistant

Figure 4. Spatially-explicit simulations of SGD and FAMSS biocontrol strategies in the context of genetic resistance. (a) Key showing location of populations treated with GE biocontrol strain. Each square represents a separate simulation model. Arrows show allowable migration routes from a single population at each time step. (b) Time-course data from a single population treated with SGD mosquitoes showing numbers of Ae. aegypti with genotypes represented in the inset legend. This model reflects data from the population in row 5, column 4 of the SGD simulation with $F_{N H E J}=10^{-3}$. (c) Performance of SGD in spatially explicit simulation with different rates of NHEJ (vertical axis). Plots show number of wild-type and resistant adult females (left, various time steps), and percentage of the final population comprising SGD mosquitoes (center, blue) or genetically resistant mosquitoes (right, red). (d) Performance of FAMSS in spatially explicit simulation with different rates of promoter conversion (vertical axis). Graphs show analogous data as (c), but with blue representing the final population of FAMSS mosquitoes. 